\title{
Constraining Lorentz Invariance Violation using the muon content of extensive air showers measured at the Pierre Auger Observatory
}

\author{
Caterina Trimarelli ${ }^{a * *}$ on behalf of the Pierre Auger ${ }^{b}$ Collaboration \\ (a complete list of authors can be found at the end of the proceedings) \\ ${ }^{a}$ Università dell'Aquila and INFN Laboratori Nazionali del Gran Sasso, Assergi (L'Aquila), Italy \\ ${ }^{b}$ Observatorio Pierre Auger, Av. San Martín Norte 304, 5613 Malargüe, Argentina \\ E-mail: spokespersons@auger.org
}

Lorentz symmetry requires the space-time structure to be the same for all observers, but, on the other hand, various quantum gravity theories suggest that it may be violated when approaching the Planck scale. Even a small violation of Lorentz Invariance (LI) could easily affect the propagation of Ultra-High Energy Cosmic Rays (UHECRs) on a cosmological scale. Moreover, at extreme energies, like those available in the collision of UHECRs with the atmosphere, one should also expect a change in the interactions due to Lorentz Invariance Violation (LIV). In this work, the effects of LIV on the development of Extensive Air Showers (EAS) have been considered. After having introduced LIV as a perturbation term in the single-particle dispersion relation, a library of simulated showers with different energies, primary particles and LIV strengths has been produced. For the first time, this effect has been studied using the muon content of air showers measured at the Pierre Auger Observatory. Limits on LIV parameters have been derived from a comparison between the Monte Carlo expectations and the muon fluctuation measurements from the Pierre Auger Observatory. The results are presented in this contribution.

$37^{\text {th }}$ International Cosmic Ray Conference (ICRC 2021)

July 12th - 23rd, 2021

Online - Berlin, Germany

\footnotetext{
${ }^{*}$ Presenter
} 


\section{Introduction}

Violations of Lorentz Invariance could affect the energy threshold of photo-hadronic interactions; in particular, depending on the composition of the UHECRs at the highest energies, the attenuation length of photo-meson production or photo-disintegration may become extremely large and suppress particle interaction during propagation in the extragalactic space [1-4]. As a consequence, the existing evidence of the suppression of the flux at the highest energies [5] can be used to put a limit on LIV. In particular, LIV can be tested by searching the best description of the UHECR observables, under LIV assumptions, as already done for instance in [6-10]. However, the scenario is complicated by the fact that the best description of the UHECR spectrum and composition is found corresponding to values of maximum energy at the source smaller than or comparable to the typical threshold energy for photo-meson or photo-disintegration reactions [11]. For this reason, the sensitivity of the deviations from LI in UHECR propagation is smaller than expected, and alternative approaches need to be investigated.

\section{Lorentz Invariance Violation framework}

One possibility to constrain LIV models is that, depending on the strength of the violation, the high energy available in the collision of cosmic rays with the atmosphere ${ }^{1}$ can lead to modifications of the shower development with respect to the standard LI case. A well established phenomenological approach to introduce LIV effects $[12,13]$ consists of adding effective terms in the dispersion relation of particles as:

$$
E^{2}-p^{2}=m^{2}+f\left(\vec{p}, M_{\mathrm{P} 1} ; \eta\right)
$$

where $m$ is the particle mass at rest, $E$ its energy, and $f$ represents the contribution of violation due to the quantum gravity effects. In this approach the violation depends on the momentum of the particle $\vec{p}$ and on the Planck mass $M_{\mathrm{Pl}}$ through the LIV parameter $\eta$, a dimensionless constant coefficient to be constrained. At $p \ll M_{\mathrm{Pl}}$, the factor $f$ can be expanded and considering only the leading order of the expansion, Eq. 1 becomes:

$$
E^{2}-p^{2}=m^{2}+\eta^{(n)} \frac{p^{n+2}}{M_{\mathrm{Pl}}^{n}}
$$

Previous works found limits to the LIV parameter by studying the effects of Lorentz invariance violation on cosmic ray propagation at first order $\eta_{\gamma}^{(1)}>-1.2 \cdot 10^{-10}[14]$ in the photon sector in the astrophysical scenario, which best describes UHECR data, and at second order $-10^{-3}<\eta_{\pi}^{(2)}<$ $10^{-1}$ [2] in the case of the neutral pion decay. Interpreting the right-hand side of the Eq. 2 as an energy dependent mass, $m_{\mathrm{LIV}}^{2}=m^{2}+\eta^{(n)} \frac{p^{n+2}}{M_{\mathrm{Pl}}^{n}}$, the Lorentz factor for a LI violating particle at energy $E$ can be defined as:

$$
\gamma_{\text {LIV }}=E / m_{\text {LIV }}
$$

Depending on the value assumed by $\eta^{(n)}$, the lifetime of the considered particle $\tau=\gamma_{L I V} \tau_{0}$ will change accordingly. For negative/positive values of $\eta^{(n)}$ the lifetime of the particle should

\footnotetext{
${ }^{1} \mathrm{As}$ an example a proton at $10^{19} \mathrm{eV}$ hitting the atmosphere corresponds to a $0.1 \mathrm{PeV}$ collision in the center of mass frame.
} 


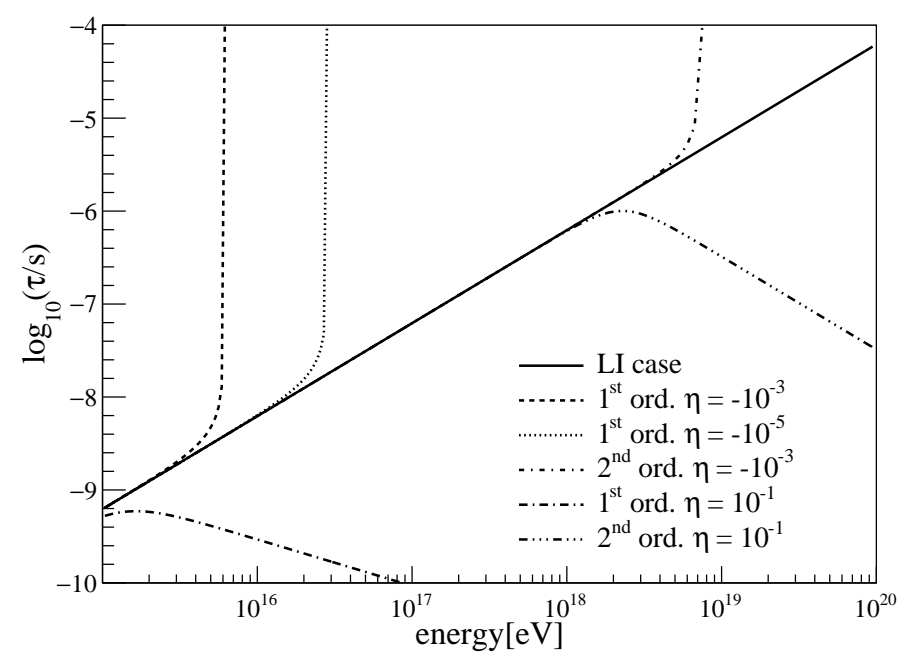

Figure 1: Neutral pion mean lifetime as a function of energy for the Lorentz invariant case and for different strengths of LIV.

increase/decrease with respect to the LI case producing modifications in the EAS development which depends both on the energy and the strength of the violation. As an example, the $\pi^{0}$ lifetime as a function of the energy for the standard case and for different values of the LIV parameters is shown in Fig. 1. For negative values of $\eta$, the mean lifetime increases up to a critical energy, corresponding to the point at which the phase space reduces to zero (i.e. $m_{\mathrm{LIV}}^{2} \rightarrow 0$ ) and the particle becomes stable (i.e. $\gamma \rightarrow \infty$ ). The energy at which the lifetime evolution deviates from the standard LI case depends both on the order and the strength of the violation. To have a qualitative idea of what one should expect, let us consider the simple model [15] where a primary hadron interacting in the atmosphere produces $2 / 3$ of charged pions $\pi^{ \pm}$and $1 / 3$ of $\pi^{0} s$. In the standard case, charged pions decay producing muons and neutrinos while the neutral ones suddenly decay in two photons producing an electromagnetic sub-shower. Otherwise, in the presence of LIV and for negative values of $\eta^{(n)}$, the $\pi^{0}$ lifetime grows and the probability to interact before decaying increases. The re-interacting $\pi^{0} \mathrm{~s}$ will behave as the source of a hadronic sub-showers like those initiated by the primary cosmic ray particle. As the energy decreases in the further shower generations, $\pi^{0} \mathrm{~s}$ will start again to produce a standard electromagnetic sub-shower. The consequence is a modification of the shower development in the atmosphere. The amount of energy deposited in the atmosphere will be reduced (i.e. invisible energy going to neutrinos will grow) leading to an underestimation of the primary energy if the event is treated as a standard physics one. Moreover, the position of the shower maximum $\left(X_{\max }\right)[16]$ will be slightly modified with respect to the standard LI case. In addition, as the muon content correlates with the energy of the hadronic component of the shower, we can predict that the number of muons produced in the EAS will increase and the physical fluctuations will decrease, as almost all the energy is kept into the hadronic component after the first stages of the shower developement, with little room for stochastic leakage to the electromagnetic component [17]. On the other hand, for positive values of the $\eta$ parameter the lifetime becomes smaller with respect to the standard LI case as the energy increases. In these cases, the lifetime 
decreases above the critical energy and the pions decay faster than in the standard one and no effect on the shower development is expected. For this reason, in this work only the effects due to negative values of the $\eta$ parameter are considered. Our purpose is to find only the lower limit of the bound for LIV at first order.

To quantify the effect of LIV on the shower development, we have performed detailed simulations of the longitudinal profile by using CONEX software $[18,19]$ in both LI and LIV cases. For the violated scenario, the software has been modified by changing the lifetimes of all the unstable particles according to Eq. 3. In particular, in the velocity definition $\beta=\frac{\mathbf{P}}{m \gamma}$, the Lorentz factor $\gamma$ has been replaced with the LIV expression $\gamma_{\text {LIV }}=E / m_{\text {LIV }}$. The values of $\eta^{(n)}$ considered $^{2}$ for this study are $-10^{-1},-10^{-2},-10^{-3},-10^{-4},-10^{-5},-10^{-6},-10^{-7},-10^{-8}$ and order of the violation $n=1,2$. For each value of $\eta, 5000$ primary cosmic ray particles have been produced in the energy range between $10^{16} \mathrm{eV}$ and $10^{21} \mathrm{eV}$, using EPOS LHC [20] and QGSJETII-04 [21] hadronic interaction models and for different primary particle types i.e. proton, helium, nitrogen, silicon and iron.

\section{Lorentz Invariance Violation effects on air shower development: Muon content distribution}

As a result of the modified air shower simulation in the presence of LIV at first order, we have considered the mean longitudinal profile $d E / d x$. For larger values of $|\eta|$ (only negative LIV parameters are taken into account) the effects due to the violation increase. In fact, we have found a shift of $X_{\max }$, and a reduction in the height of the maximum energy deposit in the atmosphere.

First of all, the displacement in the position of the maximum of the longitudinal profile leads to different values of $X_{\max }$. The change in the energy threshold of particle decays (mainly neutral pions), depleting the electromagnetic part of the shower faster, generates the effect to move the shower maximum to higher altitudes. This is due to the fact that a proton from the point of view of the shower development is behaving like a heavier nucleus. In fact, in the presence of LIV, the measured mass composition corresponds to a greater fraction of protons at the highest energies. This result has been already used in order to constrain LIV models in a previous work [22] and it is not considered in this contribution.

On the other hand, the reduction in the normalization of the longitudinal profile is linked to a change in the number of muons at the ground. In particular, the calorimetric energy deposited in the atmosphere in the presence of LIV is lower than the standard one. The modification of the energy-momentum relation allows hadronic interactions of neutral pions that contribute to the growth of the hadronic cascade producing an increase in the number of muons, as shown in Fig. 2(a), where the average number of muons at ground as a function of the primary energy in LI and LIV cases are shown. For proton-induced air showers, the number of muons is considerably increased, in any of the LIV cases considered here, while for iron primaries the effect is in general milder.

These two main effects, caused by the LIV framework, affect the fluctuations of the number of muons. In fact, the ratio of the fluctuations to the average number of muons (hereinafter referred to as relative fluctuations), dominated mostly by the first interaction [17], considerably decreases in

\footnotetext{
${ }^{2}$ Positive values of LIV parameter have been also considered but no effect on the shower development has been found.
} 
the presence of LIV, as shown in Fig. 2(b), where the relative fluctuations of the number of muons as a function of the primary energy are reported.

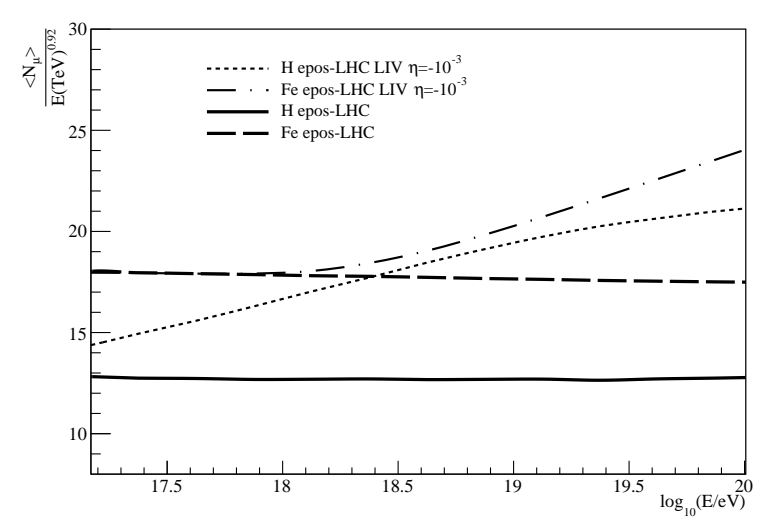

(a)

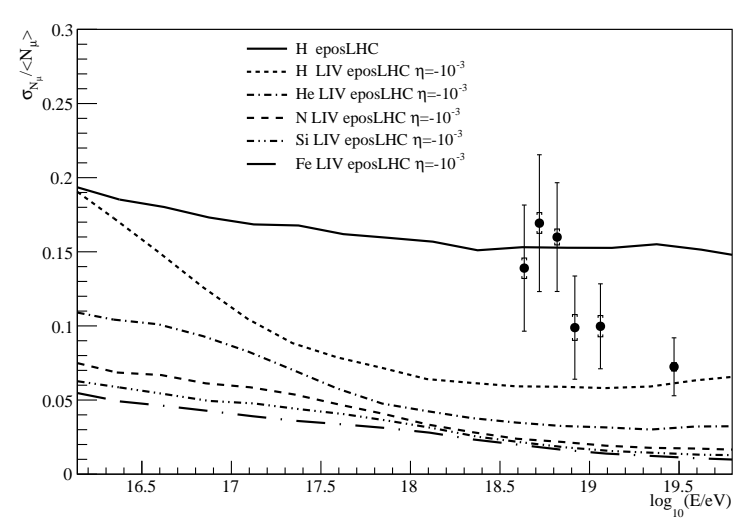

(b)

Figure 2: (a) Average number of muons at ground vs primary energy in LI and LIV cases; (b) Data (black points with error bars) compared to LI and LIV models for the relative fluctuation of the number of muons vs primary energy. The LIV case corresponds to $\eta=-10^{-3}$. The statistical uncertainty is indicated by the error bars. The total systematic uncertainty is indicated by the square brackets.

Limits on LIV parameter $\eta$ can be derived comparing the observed strong decrease of the relative fluctuations with the muon fluctuation measurement [23] from the Pierre Auger Observatory [24].

\section{Results and Discussion}

Considering the dependence of the decrease of the relative fluctuations on the different LIV strengths, a new bound for the LIV parameter $\eta^{(1)}$ has been obtained. To find this, the most conservative LIV model with respect to data, depending on the particular mixture of primary particles, should be considered. In particular, the effects of different composition scenarios on both fluctuations and average number of muons have been taken into account. It can be noticed, referring to Fig. 3 in [23], that the mixture of the two components, $p$ and $F e$, gives the maximum value of relative fluctuations. Therefore, the most conservative LIV model corresponds to the ratio of the fluctuations to the average number of muons for a mixture of proton and iron.

First of all, we have considered the following expressions for the average number of muons $\left\langle N_{\mu}\right\rangle_{\text {mix }}$ and the fluctuations $R M S D_{\text {mix }}\left(N_{\mu}\right)$ for a mixture of $p$ and $F e$ :

$$
\begin{aligned}
\left\langle N_{\mu}\right\rangle_{\text {mix }}(\alpha ; \eta) & =(1-\alpha)\left\langle N_{\mu}\right\rangle_{p}+\alpha\left\langle N_{\mu}\right\rangle_{F e} \\
R M S D_{\text {mix }}^{2}\left(N_{\mu}\right)(\alpha ; \eta) & =(1-\alpha) R M S D^{2}\left(N_{\mu}\right)_{p}+\alpha R M S D^{2}\left(N_{\mu}\right)_{F e}+\alpha(1-\alpha)\left(\left\langle N_{\mu}\right\rangle_{p}-\left\langle N_{\mu}\right\rangle_{F e}\right)^{2}
\end{aligned}
$$

where $\alpha$, which depends on the energy, is the relative abundance of iron nuclei and the subscripts $p$ and $\mathrm{Fe}$ label the averages and $R M S D$ of pure proton and iron primaries respectively ${ }^{3}$. The

${ }^{3}\left\langle N_{\mu}\right\rangle_{p},\left\langle N_{\mu}\right\rangle_{F e}, R M S D_{p}^{2}$ and $R M S D_{F e}^{2}$ depend on energy and on LIV parameter $\eta$ 


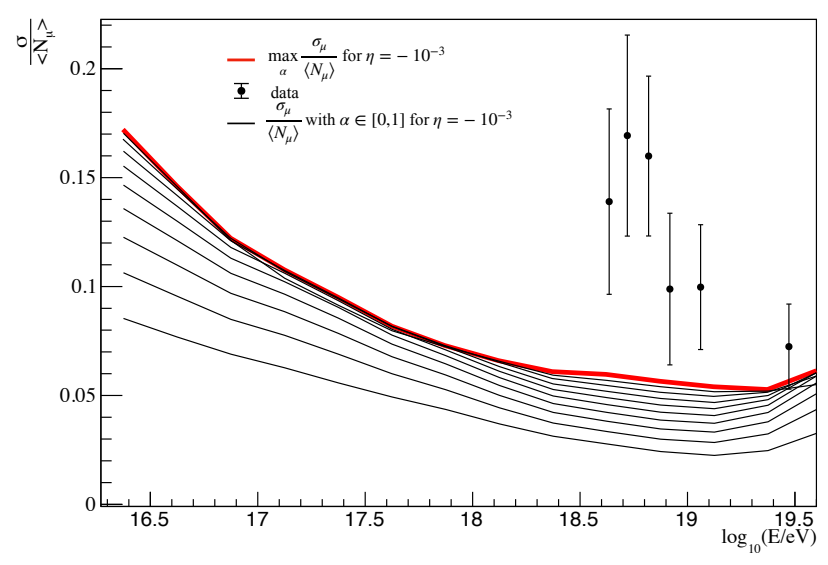

(a)

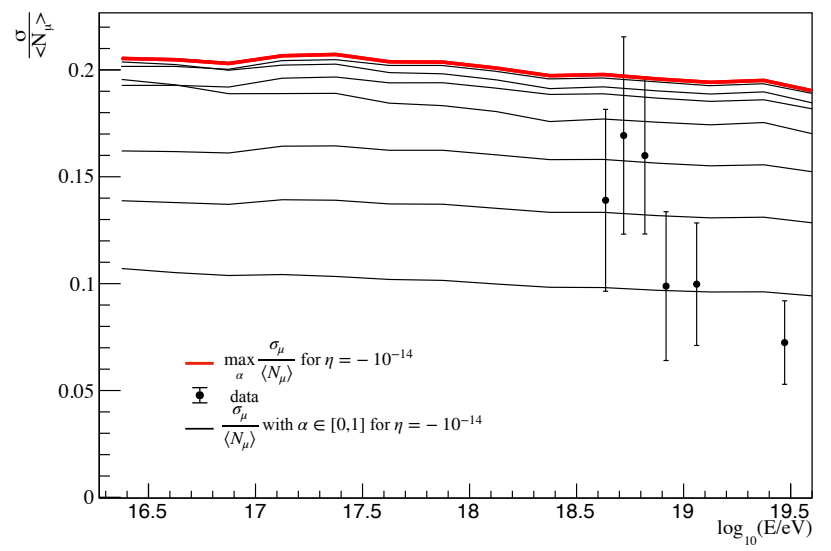

(b)

Figure 3: Relative fluctuation of the number of muons for different mixed initial proton-iron composition vs primary energy in the presence of LIV with $\eta=-10^{-3}$ (a) and $\eta=-10^{-14}$ (b). The black points with error bars represent the meausured relative fluctuations in the number of muons.

average number of muons $\left\langle N_{\mu}\right\rangle$ and the $R M S D^{2}$ for air showers induced by both proton and iron nuclei have been retrieved from the air shower simulations for six different LIV parameter values $\left(\eta^{(1)}=-10^{-3},-10^{4} .-10^{-5},-10^{-6},-10^{-7},-10^{-8}\right)$ in the energy range reported in Sec. 2. These values have been used to parametrize $\left\langle N_{\mu}\right\rangle$ and the $R M S D$ between $\log (E / \mathrm{eV})=10^{16.5}$ and $\log (E / \mathrm{eV})=10^{20}$, for $-10^{-3}<\eta<-10^{-15}$ and for different masses. For each value of $\eta$, the parameterizations can be used to calculate the expected relative fluctuations for a certain mixture of proton and iron nuclei (hereinafter referred to as mixed relative fluctuations) as:

$$
\frac{\sigma_{\mu}}{\left\langle N_{\mu}\right\rangle}(\alpha ; \eta)=\frac{\sqrt{R M S D_{\text {mix }}^{2}\left(N_{\mu}\right)(\alpha ; \eta)}}{\left\langle N_{\mu}\right\rangle_{\text {mix }}(\alpha ; \eta)}
$$

In Fig. 3, the result of the mixed relative fluctuations for different mixtures of proton-iron composition and for a fixed LIV parameter $\eta=-10^{-3}$ (3(a)) and $\eta=-10^{-14}$ (3(b)) are shown as a function of the energy. In particular, all the black curves represent the mixed relative fluctuations for a scan of the relative abundance $\alpha$ between 0 and 1, while the red curve corresponds to the maximum with respect to $\alpha$. It can be noticed that, in both 3(a) and 3(b), the red curve is above all the black ones. This effect has been observed for all the violation strengths. Therefore, only if all the curves are below the data points, the maximum with respect to $\alpha(E)$ of the Eq. 5 in each energy bin corresponds to the most conservative LIV model. For any LIV parameter value, the most conservative LIV relative fluctuations as a function of the energy can be found without repeating any shower simulation. In Fig. 4, the coloured thin curves represent the maxima with respect to $\alpha$ for the relative fluctuations obtained from the parameterizations considering the $\eta$ parameter in the range $\left[-10^{-3},-10^{-15}\right]$. Considering only the curves that are below the data, the $\chi^{2}$ can be calculated as a function of $\eta$ using each resulting mixed fluctuation and all the experimental data points. In this way, a continuous confidence level to exclude the LIV model has been found. This continuous result allows to determine the strictest lower $\eta$ parameter bound. For example, in Fig. 4 , 


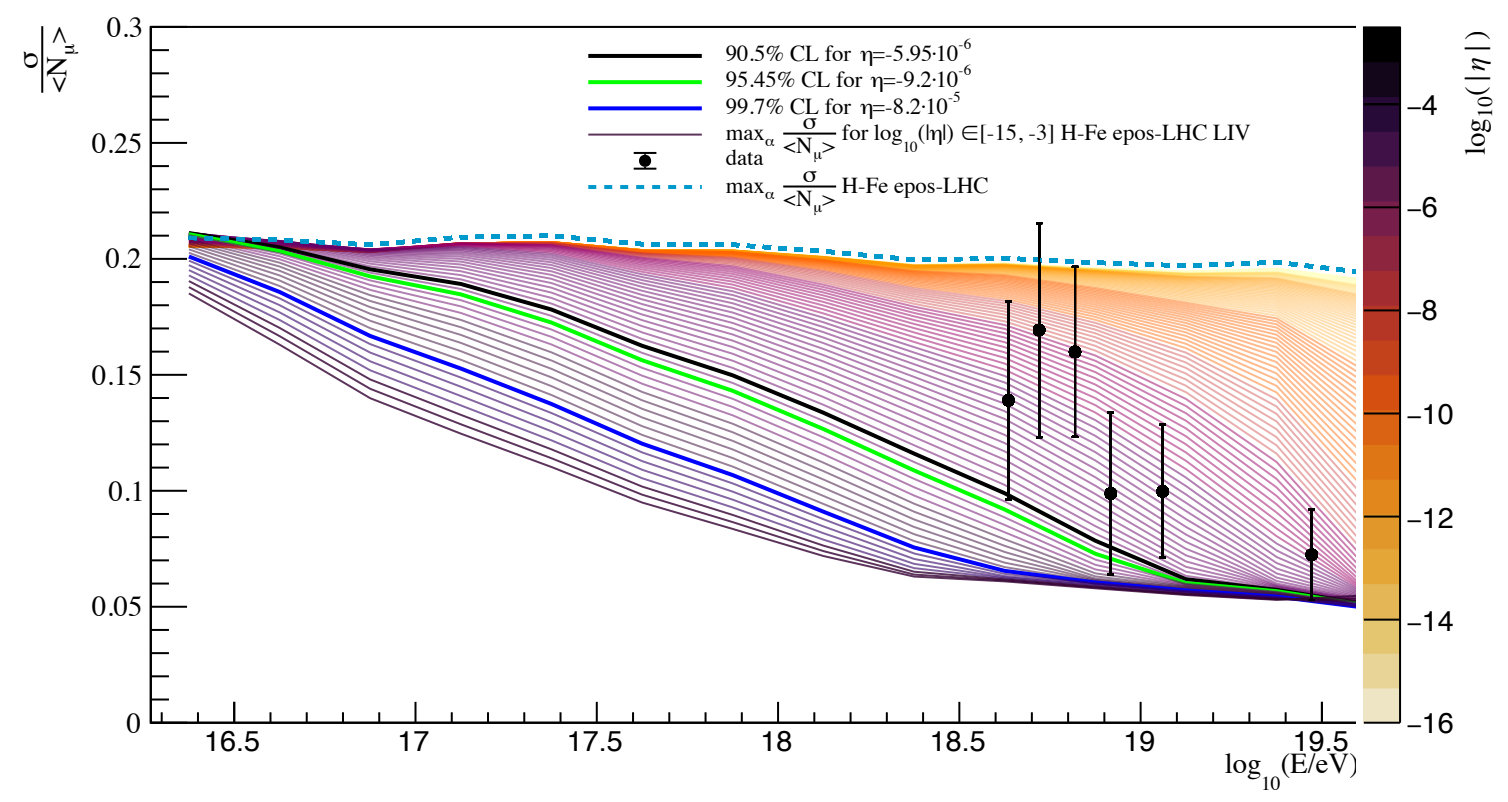

Figure 4: Maximum with respect to $\alpha$ of the mixed relative fluctuations obtained using the parameterizations in the standard case (dashed curve) and in the presence of LIV considering $\eta$ in the range $\left[-10^{-3},-10^{-15}\right]$ (coloured curves) as a function of the primary energy. Each color corresponds to a different violation strength (right axis). The black points with error bars (statistical uncertainties) represent the measured relative fluctuations in the number of muons.

the mixed relative fluctuations at three different CLs obtained considering all the experimental data are highlighted. The blue curve, corresponding to $\eta=-8.2 \cdot 10^{-5}$, refers to $99.7 \% \mathrm{CL}$. The green (black) one corresponds to $95.45 \%(90.5 \%)$ with a LIV parameter $\eta=-9.2 \cdot 10^{-6}\left(\eta=-5.95 \cdot 10^{-6}\right)$. As a consequence, the new bound for $\eta^{(1)}$ is $\left[-5.95 \cdot 10^{-6}, 10^{-1}\right]$ at $90.5 \%$ of CL.

It can be noticed that if the discrepancy in the reconstruction of the energy in the presence of LIV and the one in the standard scenario was included, a net shift of the experimental data towards the higher energies would be observed. However, this bias between the primary energy estimated if the events are treated in LIV case and in standard one is lower than the 5\% for all the considered $\eta$ parameters and, if implemented, it would lead to a further improvement of the parameter bound.

In conclusion, we have found a new lower bound of the $\eta$ parameter range of values using the maximum relative fluctuation for a mixed initial proton-iron composition for LIV at first order. In particular, we have obtained $\eta^{(1)}>-5.95 \cdot 10^{-6}$ at $90.5 \%$ of CL. A similar approach using the minimum of the relative fluctuation with respect to $\alpha$ could lead to the definition of a negative upper bound of the LIV parameter. Previous works found limits to the LIV parameter at first order by studying the effects of Lorentz invariance violation on the photon propagation in the universe [14].

Future prospects will provide for an extension of the overall procedure to the effects produced by LIV at second order. Moreover, limits on $\eta$ parameter could be found through a combined analysis considering simultaneously the relative fluctuations of the number of muons and the mass composition derived from the $X_{\max }$ measurements given by the Pierre Auger Observatory. 


\section{References}

[1] R. Aloisio et al. Phys. Rev. D 62 (2000) 053010, [astro-ph/0001258].

[2] L. Maccione et al. JCAP 04 (2009) 022, [0902 . 1756].

[3] A. Saveliev, L. Maccione and G. Sigl, JCAP 03 (2011) 046, [1101.2903].

[4] L. Maccione, A. Saveliev and G. Sigl, Proc. 12th Int. Conf. Astroparticle and Underground Physics, Munich, Germany (2011), PoS(TAUP2011) 375.

[5] A. Aab et al. [Pierre Auger Coll.], The Pierre Auger Observatory: Contributions to the 36th International Cosmic Ray Conference: Madison, Wisconsin, USA(2019), Compendium of PoS(ICRC2019), [1909. 09073].

[6] X. Bi, L. Cao, Z. Cao, L. Qiang, Y. Qiang, Y. Qiang, Phys. Rev. D 79 (2009) 083015, [0812 . 0121].

[7] S. T. Scully and F. W. Stecker, Astropart. Phys. 31 (2009) 220-225, [0811.2230].

[8] D. Boncioli et al. [for the Pierre Auger Coll.], Proc. 34th Int. Cosmic Ray Conf., The Hague, The Netherlands (2015), PoS(ICRC2015)521, [1509.03732].

[9] D. Boncioli [for the Pierre Auger Coll.], Proc. 35th Int. Cosmic Ray Conf., Busan, Korea (2017), PoS(ICRC2017)561, [1708.06592].

[10] R. G. Lang [for the Pierre Auger Coll.], Proc. 36th Int. Cosmic Ray Conf., Madison, Wisconsin, USA (2019), PoS(ICRC2019)327, [1909.09073].

[11] A. Aab et al. [Pierre Auger Coll.], JCAP 03 (2018) E02, [1612 . 07155].

[12] S. R. Coleman and S. L. Glashow, Phys. Rev. D 59 (1999) 116008, [hep-ph/9812418].

[13] H. Martínez-Huerta and A. Pérez-Lorenzana, Phys. Rev. D 95 (2017) 063001, [1610.00047].

[14] R. G. Lang, H. Martínez-Huerta and V. de Souza, Astrophys. J. 853 (2018) 23, [1701. 04865].

[15] J. Matthews, Astropart. Phys. 22 (2005) 387-397.

[16] J. Linsley and A. A. Watson, Phys. Rev. Lett. 46 (1981) 459-463.

[17] L. Cazon, R. Conceição and F. Riehn, Phys. Lett. B 784 (2018) 68-76, [1803. 05699].

[18] T. Pierog et al. Nucl. Phys. B Proc. Suppl. 151 (2006) 159-162, [astro-ph/0411260].

[19] T. Bergmann et al. Astropart. Phys. 26 (2007) 420-432, [astro-ph/0606564].

[20] T. Pierog, I. Karpenko, J. M. Katzy, E. Yatsenko and K. Werner, Phys. Rev. C 92 (2015) 034906, [1306. 0121$].$

[21] S. Ostapchenko, Phys. Rev. D 83 (2011) 014018, [1010. 1869].

[22] F. R. Klinkhamer, M. Niechciol and M. Risse, Phys. Rev. D 96 (2017) 116011, [1710. 02507].

[23] A. Aab et al. [Pierre Auger Coll.], Phys. Rev. Lett. 126 (2021) 152002, [2102 . 07797].

[24] A. Aab et al. [Pierre Auger Coll.], Nucl. Instrum. Meth. A 798 (2015) 172-213, [1502.01323]. 


\section{The Pierre Auger Collaboration}

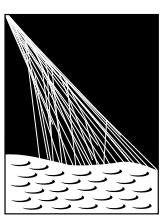

PIERRE

AUSGERVATORY

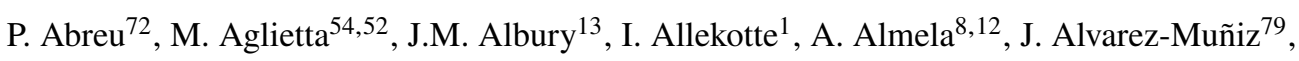
R. Alves Batista ${ }^{80}$, G.A. Anastasi ${ }^{63,52}$, L. Anchordoqui ${ }^{87}$, B. Andrada ${ }^{8}$, S. Andringa ${ }^{72}$, C. $\mathrm{Aramo}^{50}$, P.R. Araújo Ferreira ${ }^{42}$, J. C. Arteaga Velázquez ${ }^{67}$, H. Asorey $^{8}$, P. Assis ${ }^{72}$, G. Avila ${ }^{11}$, A.M. Badescu ${ }^{75}$, A. Bakalova ${ }^{32}$, A. Balaceanu ${ }^{73}$, F. Barbato ${ }^{45,46}$, R.J. Barreira Luz $^{72}$, K.H. Becker ${ }^{38}$, J.A. Bellido ${ }^{13,69}$, C. Berat ${ }^{36}$, M.E. Bertaina ${ }^{63,52}$, X. Bertou ${ }^{1}$, P.L. Biermann ${ }^{b}$, V. Binet ${ }^{6}$, K. Bismark ${ }^{39,8}$, T. Bister ${ }^{42}$, J. Biteau ${ }^{37}$, J. Blazek ${ }^{32}$, C. Bleve ${ }^{36}$, M. Boháčová ${ }^{32}$, D. Boncioli ${ }^{57,46}$, C. Bonifazi ${ }^{9,26}$, L. Bonneau Arbeletche ${ }^{21}$, N. Borodai ${ }^{70}$, A.M. Botti ${ }^{8}$, J. Brack ${ }^{d}$, T. Bretz ${ }^{42}$, P.G. Brichetto Orchera ${ }^{8}$, F.L. Briechle ${ }^{42}$, P. Buchholz ${ }^{44}$, A. Bueno ${ }^{78}$, S. Buitink ${ }^{15}$, M. Buscemi ${ }^{47}$, M. Büsken ${ }^{39,8}$, K.S. Caballero-Mora ${ }^{66}$, L. Caccianiga ${ }^{59,49}$, F. Canfora ${ }^{80,81}$, I. Caracas ${ }^{38}$, J.M. Carceller ${ }^{78}$, R. Caruso ${ }^{58,47}$, A. Castellina ${ }^{54,52}$, F. Catalani ${ }^{19}$, G. Cataldi ${ }^{48}$, L. Cazon ${ }^{72}$, M. Cerda ${ }^{10}$, J.A. Chinellato ${ }^{22}$, J. Chudoba ${ }^{32}$, L. Chytka ${ }^{33}$, R.W. Clay ${ }^{13}$, A.C. Cobos Ceruttii ${ }^{7}$, R. Colalillo ${ }^{60,50}$, A. Coleman ${ }^{93}$, M.R. Coluccia ${ }^{48}$, R. Conceição ${ }^{72}$, A. Condorelli ${ }^{45,46}$, G. Consolati ${ }^{49,55}$, F. Contreras ${ }^{11}$, F. Convenga ${ }^{56,48}$, D. Correia dos Santos $^{28}$, C.E. Covault ${ }^{85}$, S. Dasso ${ }^{5,3}$, K. Daumiller ${ }^{41}$, B.R. Dawson ${ }^{13}$, J.A. Day ${ }^{13}$, R.M. de Almeida $^{28}$, J. de Jesús ${ }^{8,41}$, S.J. de Jong ${ }^{80,81}$, G. De Mauro ${ }^{80,81}$, J.R.T. de Mello Neto ${ }^{26,27}$, I. De Mitri ${ }^{45,46}$, J. de Oliveira ${ }^{18}$, D. de Oliveira Franco ${ }^{22}$, F. de Palma ${ }^{56,48}$, V. de Souza $^{20}$, E. De Vito ${ }^{56,48}$, M. del Río ${ }^{11}$, O. Deligny ${ }^{34}$, L. Deval ${ }^{41,8}$, A. di Matteo $^{52}$, C. Dobrigkeit ${ }^{22}$, J.C. D’Olivo ${ }^{68}$, L.M. Domingues Mendes ${ }^{72}$, R.C. dos Anjos ${ }^{25}$, D. dos Santos $^{28}$, M.T. Dova ${ }^{4}$, J. Ebr ${ }^{32}$, R. Engel ${ }^{39,41}$, I. Epicoco ${ }^{56,48}$, M. Erdmann ${ }^{42}$, C.O. Escobar ${ }^{a}$, A. Etchegoyen ${ }^{8,12}$, H. Falcke ${ }^{80,82,81}$, J. Farmer ${ }^{92}$, G. Farrar ${ }^{90}$, A.C. Fauth ${ }^{22}$, N. Fazzini ${ }^{a}$, F. Feldbusch ${ }^{40}$, F. Fenu ${ }^{54,52}$,

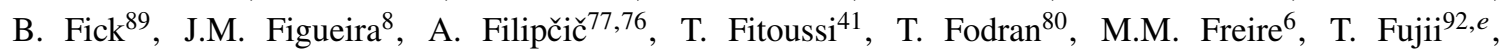
A. Fuster $^{8,12}$, C. Galea ${ }^{80}$, C. Galelli ${ }^{59,49}$, B. García ${ }^{7}$, A.L. Garcia Vegas ${ }^{42}$, H. Gemmeke ${ }^{40}$, F. Gesualdi ${ }^{8,41}$, A. Gherghel-Lascu ${ }^{73}$, P.L. Ghia ${ }^{34}$, U. Giaccari ${ }^{80}$, M. Giammarchi ${ }^{49}$, J. Glombitza ${ }^{42}$, F. Gobbi ${ }^{10}$, F. Gollan ${ }^{8}$, G. Golup ${ }^{1}$, M. Gómez Berisso ${ }^{1}$, P.F. Gómez Vitale ${ }^{11}$, J.P. Gongora ${ }^{11}$, J.M. González ${ }^{1}$, N. González ${ }^{14}$, I. Goos ${ }^{1,41}$, D. Góra ${ }^{70}$, A. Gorgi ${ }^{54,52}$, M. Gottowik ${ }^{38}$, T.D. Grubb ${ }^{13}$, F. Guarino ${ }^{60,50}$, G.P. Guedes ${ }^{23}$, E. Guido ${ }^{52,63}$, S. $\mathrm{Hahn}^{41,8}$, P. $\mathrm{Hamal}^{32}$, M.R. Hampel ${ }^{8}$, P. Hansen ${ }^{4}$, D. Harari ${ }^{1}$, V.M. Harvey ${ }^{13}$, A. Haungs ${ }^{41}$, T. Hebbeker ${ }^{42}$, D. Heck ${ }^{41}$, G.C. Hill ${ }^{13}$, C. Hojvat ${ }^{a}$, J.R. Hörandel ${ }^{80,81}$, P. Horvath ${ }^{33}$, M. Hrabovský ${ }^{33}$, T. Huege ${ }^{41,15}$, A. Insolia ${ }^{58,47}$, P.G. Isar $^{74}$, P. Janecek ${ }^{32}$, J.A. Johnsen ${ }^{86}$, J. Jurysek ${ }^{32}$, A. Kääpä ${ }^{38}$, K.H. Kampert ${ }^{38}$, N. Karastathis ${ }^{41}$, B. Keilhauer ${ }^{41}$, J. Kemp ${ }^{42}$, A. Khakurdikar ${ }^{80}$, V.V. Kizakke Covilakam $^{8,41}$, H.O. Klages ${ }^{41}$, M. Kleifges ${ }^{40}$, J. Kleinfeller ${ }^{10}$, M. Köpke ${ }^{39}$, N. Kunka ${ }^{40}$, B.L. Lago ${ }^{17}$, R.G. Lang ${ }^{20}$, N. Langner ${ }^{42}$, M.A. Leigui de Oliveira ${ }^{24}$, V. Lenok $^{41}$, A. Letessier-Selvon ${ }^{35}$, I. LhenryYvon $^{34}$, D. Lo Presti ${ }^{58,47}$, L. Lopes ${ }^{72}$, R. López ${ }^{64}$, L. Lu ${ }^{94}$, Q. Luce ${ }^{39}$, J.P. Lundquist ${ }^{76}$, A. Machado Payeras $^{22}$, G. Mancarella ${ }^{56,48}$, D. Mandat ${ }^{32}$, B.C. Manning ${ }^{13}$, J. Manshanden ${ }^{43}$, P. Mantsch ${ }^{a}$, S. Marafico ${ }^{34}$, A.G. Mariazzi ${ }^{4}$, I.C. Mariş ${ }^{14}$, G. Marsella ${ }^{61,47}$, D. Martello ${ }^{56,48}$, S. Martinelli ${ }^{41,8}$, O. Martínez Bravo ${ }^{64}$, M. Mastrodicasa ${ }^{57,46}$, H.J. Mathes ${ }^{41}$, J. Matthews ${ }^{88}$, G. Matthiae ${ }^{62,51}$, E. Mayotte ${ }^{38}$, P.O. Mazur ${ }^{a}$, G. MedinaTanco $^{68}$, D. Melo $^{8}$, A. Menshikov ${ }^{40}$, K.-D. Merenda ${ }^{86}$, S. Michal ${ }^{33}$, M.I. Micheletti ${ }^{6}$, L. Miramonti ${ }^{59,49}$, S. Mollerach ${ }^{1}$, F. Montanet ${ }^{36}$, C. Morello ${ }^{54,52}$, M. Mostafá ${ }^{91}$, A.L. Müller ${ }^{8}$, M.A. Muller ${ }^{22}$, K. Mulrey ${ }^{15}$, R. Mussa ${ }^{52}$, M. Muzio ${ }^{90}$, W.M. Namasaka ${ }^{38}$, A. Nasr-Esfahani ${ }^{38}$, L. Nellen ${ }^{68}$, M. Niculescu-Oglinzanu ${ }^{73}$, M. Niechciol ${ }^{44}$, D. Nitz ${ }^{89}$, D. Nosek ${ }^{31}$, V. Novotny ${ }^{31}$, L. Nožka ${ }^{33}$, A Nucita ${ }^{56,48}$, L.A. Núñez ${ }^{30}$, M. Palatka ${ }^{32}$, J. Pallotta ${ }^{2}$, P. Papenbreer ${ }^{38}$, G. Parente ${ }^{79}$, A. Parra ${ }^{64}$, J. Pawlowsky ${ }^{38}$, M. Pech ${ }^{32}$, F. Pedreira ${ }^{79}$, J. Pȩkala ${ }^{70}$, R. Pelayo ${ }^{65}$, J. Peña-Rodriguez ${ }^{30}$, E.E. Pereira Martins ${ }^{39,8}$, J. Perez Armand ${ }^{21}$, C. Pérez Bertolli $^{8,41}$, M. Perlin ${ }^{8,41}$, L. Perrone ${ }^{56,48}$, S. Petrera ${ }^{45,46}$, T. Pierog ${ }^{41}$, M. Pimenta ${ }^{72}$, V. Pirronello ${ }^{58,47}$, M. Platino ${ }^{8}$, B. Pont $^{80}$, M. Pothast ${ }^{81,80}$, P. Privitera ${ }^{92}$, M. Prouza ${ }^{32}$, A. Puyleart ${ }^{89}$, S. Querchfeld ${ }^{38}$, J. Rautenberg ${ }^{38}$, D. Ravignani ${ }^{8}$, M. Reininghaus ${ }^{41,8}$, J. Ridky ${ }^{32}$, F. Riehn ${ }^{72}$, M. Risse ${ }^{44}$, V. Rizi ${ }^{57,46}$, W. Rodrigues de Carvalho ${ }^{21}$, J. Rodriguez Rojo ${ }^{11}$, M.J. Roncoroni ${ }^{8}$, S. Rossoni ${ }^{43}$, M. Roth ${ }^{41}$, E. Roulet ${ }^{1}$, A.C. Rovero ${ }^{5}$, P. Ruehl ${ }^{44}$, A. Saftoiu ${ }^{73}$, F. Salamida ${ }^{57,46}$, H. Salazar ${ }^{64}$, G. Salina ${ }^{51}$, J.D. Sanabria Gomez ${ }^{30}$, F. Sánchez ${ }^{8}$, E.M. Santos ${ }^{21}$, E. Santos ${ }^{32}$, F. Sarazin ${ }^{86}$, R. Sarmento ${ }^{72}$, C. Sarmiento-Cano ${ }^{8}$, R. Sato ${ }^{11}$, 
P. Savina ${ }^{56,48,34,94}$, C.M. Schäfer ${ }^{41}$, V. Scherini ${ }^{56,48}$, H. Schieler ${ }^{41}$, M. Schimassek ${ }^{39,8}$, M. Schimp ${ }^{38}$, F. Schlüter ${ }^{41,8}$, D. Schmidt ${ }^{39}$, O. Scholten ${ }^{84,15}$, P. Schovánek ${ }^{32}$, F.G. Schröder ${ }^{93,41}$, S. Schröder ${ }^{38}$, J. Schulte ${ }^{42}$, S.J. Sciutto ${ }^{4}$, M. Scornavacche ${ }^{8,41}$, A. Segreto ${ }^{53,47}$, S. Sehgal ${ }^{38}$, R.C. Shellard ${ }^{16}$, G. Sigl ${ }^{43}$, G. Silli ${ }^{8,41}$, O. Sima ${ }^{73, f}$, R. Šmída ${ }^{92}$, P. Sommers ${ }^{91}$, J.F. Soriano ${ }^{87}$, J. Souchard ${ }^{36}$, R. Squartini ${ }^{10}$, M. Stadelmaier ${ }^{41,8}$, D. Stanca ${ }^{73}$, S. Stanič ${ }^{76}$, J. Stasielak ${ }^{70}$, P. Stassi ${ }^{36}$, A. Streich ${ }^{39,8}$, M. Suárez-Durán ${ }^{14}$, T. Sudholz ${ }^{13}$, T. Suomijärvi ${ }^{37}$, A.D. Supanitsky ${ }^{8}$, Z. Szadkowski ${ }^{71}$, A. Tapia ${ }^{29}$, C. Taricco ${ }^{63,52}$, C. Timmermans ${ }^{81,80}$, O. Tkachenko ${ }^{41}$, P. Tobiska ${ }^{32}$, C.J. Todero Peixoto ${ }^{19}$, B. Tomé ${ }^{72}$, Z. Torrès ${ }^{36}$, A. Travaini ${ }^{10}$, P. Travnicek $^{32}$, C. Trimarelli ${ }^{57,46}$, M. Tueros ${ }^{4}$, R. Ulrich ${ }^{41}$, M. Unger ${ }^{41}$, L. Vaclavek ${ }^{33}$, M. Vacula ${ }^{33}$, J.F. Valdés Galicia ${ }^{68}$, L. Valore ${ }^{60,50}$, E. Varela ${ }^{64}$, A. Vásquez-Ramírez ${ }^{30}$, D. Veberič ${ }^{41}$, C. Ventura ${ }^{27}$, I.D. Vergara Quispe ${ }^{4}$, V. Verzi ${ }^{51}$, J. Vicha ${ }^{32}$, J. Vink $^{83}$, S. Vorobiov ${ }^{76}$, H. Wahlberg ${ }^{4}$, C. Watanabe ${ }^{26}$, A.A. Watson ${ }^{c}$, M. Weber ${ }^{40}$, A. Weindl ${ }^{41}$, L. Wiencke ${ }^{86}$, H. Wilczyński ${ }^{70}$, M. Wirtz ${ }^{42}$, D. Wittkowski ${ }^{38}$, B. Wundheiler ${ }^{8}$, A. Yushkov $^{32}$, O. Zapparrata ${ }^{14}$, E. Zas ${ }^{79}$, D. Zavrtanik ${ }^{76,77}$, M. Zavrtanik ${ }^{77,76}$, L. Zehrer ${ }^{76}$

${ }^{1}$ Centro Atómico Bariloche and Instituto Balseiro (CNEA-UNCuyo-CONICET), San Carlos de Bariloche, Argentina

${ }^{2}$ Centro de Investigaciones en Láseres y Aplicaciones, CITEDEF and CONICET, Villa Martelli, Argentina

${ }^{3}$ Departamento de Física and Departamento de Ciencias de la Atmósfera y los Océanos, FCEyN, Universidad de Buenos Aires and CONICET, Buenos Aires, Argentina

${ }^{4}$ IFLP, Universidad Nacional de La Plata and CONICET, La Plata, Argentina

${ }^{5}$ Instituto de Astronomía y Física del Espacio (IAFE, CONICET-UBA), Buenos Aires, Argentina

${ }^{6}$ Instituto de Física de Rosario (IFIR) - CONICET/U.N.R. and Facultad de Ciencias Bioquímicas y Farmacéuticas U.N.R., Rosario, Argentina

${ }^{7}$ Instituto de Tecnologías en Detección y Astropartículas (CNEA, CONICET, UNSAM), and Universidad Tecnológica Nacional - Facultad Regional Mendoza (CONICET/CNEA), Mendoza, Argentina

${ }^{8}$ Instituto de Tecnologías en Detección y Astropartículas (CNEA, CONICET, UNSAM), Buenos Aires, Argentina

${ }^{9}$ International Center of Advanced Studies and Instituto de Ciencias Físicas, ECyT-UNSAM and CONICET, Campus Miguelete - San Martín, Buenos Aires, Argentina

${ }^{10}$ Observatorio Pierre Auger, Malargüe, Argentina

${ }^{11}$ Observatorio Pierre Auger and Comisión Nacional de Energía Atómica, Malargüe, Argentina

12 Universidad Tecnológica Nacional - Facultad Regional Buenos Aires, Buenos Aires, Argentina

${ }^{13}$ University of Adelaide, Adelaide, S.A., Australia

14 Université Libre de Bruxelles (ULB), Brussels, Belgium

15 Vrije Universiteit Brussels, Brussels, Belgium

${ }^{16}$ Centro Brasileiro de Pesquisas Fisicas, Rio de Janeiro, RJ, Brazil

${ }^{17}$ Centro Federal de Educação Tecnológica Celso Suckow da Fonseca, Nova Friburgo, Brazil

${ }^{18}$ Instituto Federal de Educação, Ciência e Tecnologia do Rio de Janeiro (IFRJ), Brazil

${ }^{19}$ Universidade de São Paulo, Escola de Engenharia de Lorena, Lorena, SP, Brazil

${ }^{20}$ Universidade de São Paulo, Instituto de Física de São Carlos, São Carlos, SP, Brazil

${ }^{21}$ Universidade de São Paulo, Instituto de Física, São Paulo, SP, Brazil

${ }^{22}$ Universidade Estadual de Campinas, IFGW, Campinas, SP, Brazil

${ }^{23}$ Universidade Estadual de Feira de Santana, Feira de Santana, Brazil

${ }^{24}$ Universidade Federal do ABC, Santo André, SP, Brazil

${ }^{25}$ Universidade Federal do Paraná, Setor Palotina, Palotina, Brazil

${ }^{26}$ Universidade Federal do Rio de Janeiro, Instituto de Física, Rio de Janeiro, RJ, Brazil

${ }^{27}$ Universidade Federal do Rio de Janeiro (UFRJ), Observatório do Valongo, Rio de Janeiro, RJ, Brazil

${ }^{28}$ Universidade Federal Fluminense, EEIMVR, Volta Redonda, RJ, Brazil

${ }^{29}$ Universidad de Medellín, Medellín, Colombia

${ }^{30}$ Universidad Industrial de Santander, Bucaramanga, Colombia

${ }^{31}$ Charles University, Faculty of Mathematics and Physics, Institute of Particle and Nuclear Physics, Prague, Czech Republic

32 Institute of Physics of the Czech Academy of Sciences, Prague, Czech Republic 
${ }^{33}$ Palacky University, RCPTM, Olomouc, Czech Republic

34 CNRS/IN2P3, IJCLab, Université Paris-Saclay, Orsay, France

${ }^{35}$ Laboratoire de Physique Nucléaire et de Hautes Energies (LPNHE), Sorbonne Université, Université de Paris, CNRSIN2P3, Paris, France

${ }^{36}$ Univ. Grenoble Alpes, CNRS, Grenoble Institute of Engineering Univ. Grenoble Alpes, LPSC-IN2P3, 38000 Grenoble, France

${ }^{37}$ Université Paris-Saclay, CNRS/IN2P3, IJCLab, Orsay, France

38 Bergische Universität Wuppertal, Department of Physics, Wuppertal, Germany

${ }^{39}$ Karlsruhe Institute of Technology (KIT), Institute for Experimental Particle Physics, Karlsruhe, Germany

${ }^{40}$ Karlsruhe Institute of Technology (KIT), Institut für Prozessdatenverarbeitung und Elektronik, Karlsruhe, Germany

${ }^{41}$ Karlsruhe Institute of Technology (KIT), Institute for Astroparticle Physics, Karlsruhe, Germany

${ }^{42}$ RWTH Aachen University, III. Physikalisches Institut A, Aachen, Germany

${ }^{43}$ Universität Hamburg, II. Institut für Theoretische Physik, Hamburg, Germany

${ }^{44}$ Universität Siegen, Department Physik - Experimentelle Teilchenphysik, Siegen, Germany

${ }^{45}$ Gran Sasso Science Institute, L'Aquila, Italy

46 INFN Laboratori Nazionali del Gran Sasso, Assergi (L'Aquila), Italy

${ }^{47}$ INFN, Sezione di Catania, Catania, Italy

${ }^{48}$ INFN, Sezione di Lecce, Lecce, Italy

${ }^{49}$ INFN, Sezione di Milano, Milano, Italy

${ }^{50}$ INFN, Sezione di Napoli, Napoli, Italy

${ }^{51}$ INFN, Sezione di Roma "Tor Vergata", Roma, Italy

52 INFN, Sezione di Torino, Torino, Italy

53 Istituto di Astrofisica Spaziale e Fisica Cosmica di Palermo (INAF), Palermo, Italy

54 Osservatorio Astrofisico di Torino (INAF), Torino, Italy

55 Politecnico di Milano, Dipartimento di Scienze e Tecnologie Aerospaziali, Milano, Italy

${ }^{56}$ Università del Salento, Dipartimento di Matematica e Fisica "E. De Giorgi”, Lecce, Italy

57 Università dell'Aquila, Dipartimento di Scienze Fisiche e Chimiche, L'Aquila, Italy

58 Università di Catania, Dipartimento di Fisica e Astronomia, Catania, Italy

${ }^{59}$ Università di Milano, Dipartimento di Fisica, Milano, Italy

${ }^{60}$ Università di Napoli "Federico II", Dipartimento di Fisica "Ettore Pancini”, Napoli, Italy

${ }^{61}$ Università di Palermo, Dipartimento di Fisica e Chimica "E. Segrè", Palermo, Italy

62 Università di Roma "Tor Vergata", Dipartimento di Fisica, Roma, Italy

${ }^{63}$ Università Torino, Dipartimento di Fisica, Torino, Italy

${ }^{64}$ Benemérita Universidad Autónoma de Puebla, Puebla, México

${ }^{65}$ Unidad Profesional Interdisciplinaria en Ingeniería y Tecnologías Avanzadas del Instituto Politécnico Nacional (UPIITA-IPN), México, D.F., México

66 Universidad Autónoma de Chiapas, Tuxtla Gutiérrez, Chiapas, México

${ }^{67}$ Universidad Michoacana de San Nicolás de Hidalgo, Morelia, Michoacán, México

${ }^{68}$ Universidad Nacional Autónoma de México, México, D.F., México

${ }^{69}$ Universidad Nacional de San Agustin de Arequipa, Facultad de Ciencias Naturales y Formales, Arequipa, Peru

${ }^{70}$ Institute of Nuclear Physics PAN, Krakow, Poland

${ }^{71}$ University of Łódź, Faculty of High-Energy Astrophysics, Łódź, Poland

${ }^{72}$ Laboratório de Instrumentação e Física Experimental de Partículas - LIP and Instituto Superior Técnico - IST, Universidade de Lisboa - UL, Lisboa, Portugal

73 "Horia Hulubei” National Institute for Physics and Nuclear Engineering, Bucharest-Magurele, Romania

${ }^{74}$ Institute of Space Science, Bucharest-Magurele, Romania

75 University Politehnica of Bucharest, Bucharest, Romania

76 Center for Astrophysics and Cosmology (CAC), University of Nova Gorica, Nova Gorica, Slovenia

${ }^{77}$ Experimental Particle Physics Department, J. Stefan Institute, Ljubljana, Slovenia

78 Universidad de Granada and C.A.F.P.E., Granada, Spain

${ }^{79}$ Instituto Galego de Física de Altas Enerxías (IGFAE), Universidade de Santiago de Compostela, Santiago de Compostela, Spain 
${ }^{80}$ IMAPP, Radboud University Nijmegen, Nijmegen, The Netherlands

${ }^{81}$ Nationaal Instituut voor Kernfysica en Hoge Energie Fysica (NIKHEF), Science Park, Amsterdam, The Netherlands

82 Stichting Astronomisch Onderzoek in Nederland (ASTRON), Dwingeloo, The Netherlands

${ }^{83}$ Universiteit van Amsterdam, Faculty of Science, Amsterdam, The Netherlands

${ }^{84}$ University of Groningen, Kapteyn Astronomical Institute, Groningen, The Netherlands

85 Case Western Reserve University, Cleveland, OH, USA

${ }^{86}$ Colorado School of Mines, Golden, CO, USA

${ }^{87}$ Department of Physics and Astronomy, Lehman College, City University of New York, Bronx, NY, USA

${ }^{88}$ Louisiana State University, Baton Rouge, LA, USA

${ }^{89}$ Michigan Technological University, Houghton, MI, USA

${ }^{90}$ New York University, New York, NY, USA

${ }^{91}$ Pennsylvania State University, University Park, PA, USA

92 University of Chicago, Enrico Fermi Institute, Chicago, IL, USA

93 University of Delaware, Department of Physics and Astronomy, Bartol Research Institute, Newark, DE, USA

${ }^{94}$ University of Wisconsin-Madison, Department of Physics and WIPAC, Madison, WI, USA

${ }^{a}$ Fermi National Accelerator Laboratory, Fermilab, Batavia, IL, USA

${ }^{b}$ Max-Planck-Institut für Radioastronomie, Bonn, Germany

${ }^{c}$ School of Physics and Astronomy, University of Leeds, Leeds, United Kingdom

${ }^{d}$ Colorado State University, Fort Collins, CO, USA

$e^{e}$ now at Hakubi Center for Advanced Research and Graduate School of Science, Kyoto University, Kyoto, Japan

$f$ also at University of Bucharest, Physics Department, Bucharest, Romania 